\section{A case of pulmonary typical carcinoid with an extensive oncocytic component showing intense uptake of FDG}

Pulmonary carcinoid tumours, especially typical carcinoid, usually have lower FDG uptake than lung carcinoma. ${ }^{1}$ We present an unusual case of pulmonary carcinoid showing intense uptake of 18F-fluoro-deoxy-glucose (FDG).

FDG-PET CT scan showed increased tracer concentration corresponding to the $3 \mathrm{~cm}$ round tumour in the left lingula (figure 1). The maximal standardised uptake value (SUV) of the

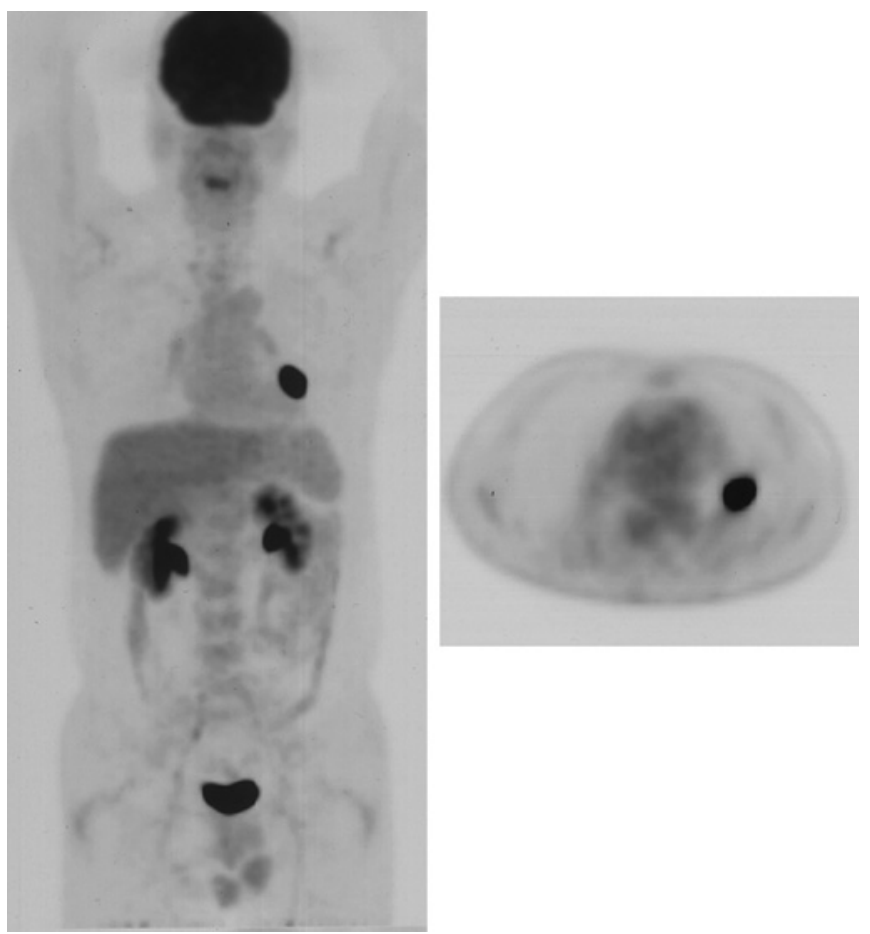

Figure 1 FDG-PET CT scan. FDG-PET CT scan showed increased tracer concentration in the left lingula, corresponding to the tumour seen on the CT scan. The maximal standardised uptake value (SUV) of the tumour was 39 .

\section{Learning points}

- This is the first case of the pulmonary typical carcinoid with an extensive oncocytic component, which showed an intense uptake of FDG.

- The extensive oncocytic component might be associated with the intense uptake of FDG.

- The positive status of Glut-1 is considered to be one of the reasons for the high uptake of FDG in the present case.

tumour was 39. Bronchoscopy was performed to show an endobronchial red-coloured polypoid mass with a smooth surface in the left $\mathrm{B}^{5} \mathrm{a}$. Cytology obtained by endobronchial brushing showed uniform round to oval nuclei with finely stippled chromatin and abundant cytoplasm. Mitotic figures were scarce. There was a diagnostic discrepancy between the findings of bronchoscopic examination and the intense uptake on FDG-PET; however, pulmonary carcinoid was tentatively diagnosed.

Left lingula resection was undertaken. The cut surface of the tumour was yellow and tumour was located in the dilated bronchus of left $\mathrm{B}^{5} \mathrm{a}$. Histopathological examination of the tumour was compatible with typical carcinoid with an extensive oncocytic component (figure $2 \mathrm{~A}, \mathrm{~B}$ ). The oncocytic type is among the most unusual type of all bronchopulmonary carcinoid. ${ }^{2}$ Moreover, the majority of oncocytic carcinoids occur as central lesions grossly indistinguishable from typical pulmonary carcinoid. $^{2}$

The oncocytic component was highly positively stained for glucose transporter protein (Glut)-1 (figure 2C), which was reported to be correlated with FDG uptake. ${ }^{3}$ The extensive oncocytic component might be associated with the intense uptake of FDG

The patient has been doing well 1 year after the operation without local recurrence and distal metastasis.

\section{Toru Kadowaki, ${ }^{1}$ Shuichi Yano, ${ }^{1}$ Kunio Araki, ${ }^{2}$ Takeshi Tokushima, ${ }^{2}$ Nobuo Morioka ${ }^{3}$}

${ }^{1}$ Division of Pulmonary Medicine, National Hospital Organization Matsue Medica Center, Matsue, Shimane, Japan; '2Division of Surgery, National Hospital Organization Matsue Medical Center, Matsue, Shimane, Japan; ${ }^{3}$ Division of Radiology, Matsue Red Cross Hospital, Matsue, Shimane, Japan
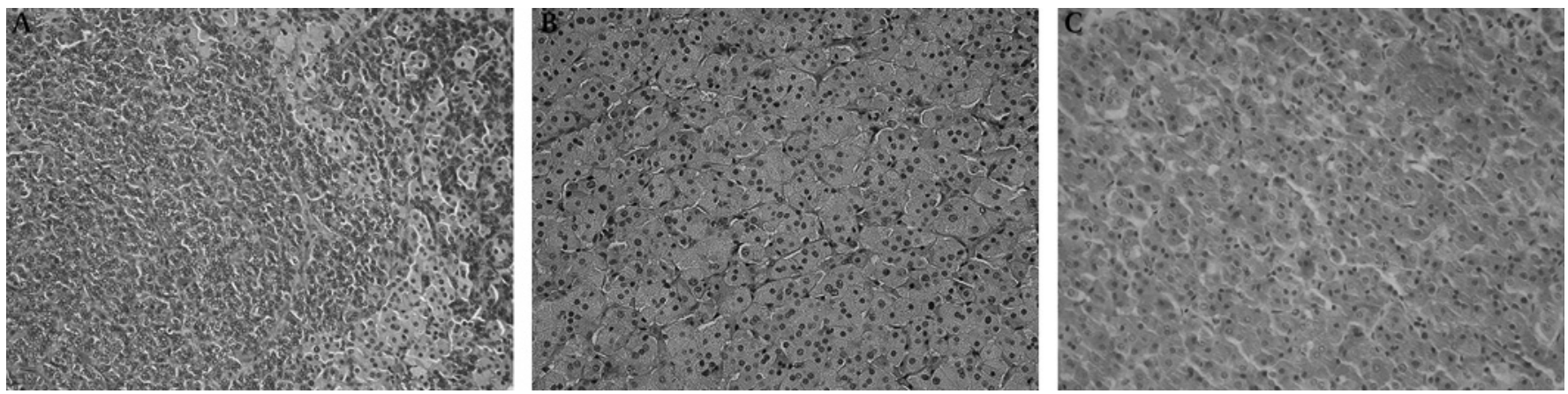

Figure 2 Histology of the resected lung tumour. (A) An area of typical carcinoid. Trabecular and mosaic architecture of uniform cells separated by a thin fibrovascular stroma showed a lower mitotic rate $(<2 / 10$ high-power field) without necrosis. (B) An area of the oncocytic component of the tumour. Adjacent to the part of typical carcinoid, there were large polygonal cells with the granular and eosinophilic cytoplasm with nuclei with finely granular chromatin. These were consistent with oncocytic carcinoid. (C) Immunohistochemical finding of oncocytic component was highly positive for Glut-1. 
Correspondence to Toru Kadowaki, Department of Pulmonary Medicine, National Hospital Organization Matsue Medical Center, 8-31, 5 cho-me, Agenogi, Matsue, Shimane 690-8556, Japan; toruyan@matsue.hosp.go.jp

Competing interests None.

Patient consent Obtained.

Provenance and peer review Not commissioned; externally peer reviewed.

Accepted 4 May 2010

Published Online First 12 November 2010

Thorax 2011:66:361-362. doi:10.1136/thx.2010.135525

\section{REFERENCES}

1. Chong S, Lee KS, Kim B-T, et al. Integrated PET/CT of pulmonary neuroendocrine tumors: diagnostic and prognostic implications. AJR 2007; 188:1223-31.

2. Arora R, Mathur SR, Aron M, et al. Oncocytic carcinoid tumor of the lung. A case report of diagnostic pitfall in filter membrane preparation of bronchial washings. Acta Cytol 2007;51:907-10.

3. Suemitsu R, Maruyama R, Nishiyama K, et al. Pulmonary typical carcinoid tumor and liver metastasis with hypermetabolism on 18-Fluorodeoxyglucose PET: A case report. Ann Thorac Cardiovasc Surg 2008;14:109-11. 